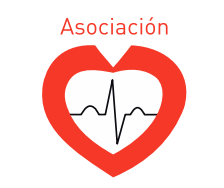

SOCIEDAD COLOMBIANA DE CARDIOLOGÍA \& CIRUGÍA CARDIOVASCULAR
Revista Colombiana de

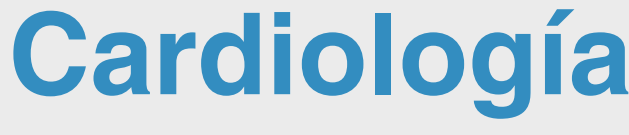

www.elsevier.es/revcolcar

ARTÍCULO ESPECIAL

\title{
Guía de práctica clínica para la prevención, el diagnóstico, el tratamiento y la rehabilitación de la falla cardiaca en población mayor de 18 años, clasificación B, C y D. Componente de rehabilitación cardiaca
}

\author{
Luz Helena Lugo-Agudelo ${ }^{a}$, Sergio Daniel Ortiz ${ }^{a}$, Andrés Mauricio Rangel ${ }^{b}$, \\ Andrés Ospina ${ }^{c}$ y Juan M. Sénior ${ }^{\mathrm{d}, e, f, *}$ \\ a Grupo de Rehabilitación en Salud, Universidad de Antioquia, Medellín, Colombia \\ ${ }^{b}$ Universidad de Antioquia, Medellín, Colombia \\ c Clínica Las Américas, Medellín, Colombia \\ d Posgrado de Cardiología Clínica y Cardiología Intervencionista, Universidad de Antioquia, Medellín, Colombia \\ e Hospital Universitario San Vicente Fundación, Medellín, Colombia \\ ${ }^{f}$ Grupo para el Estudio de las Enfermedades Cardiovasculares, Universidad de Antioquia, Medellín, Colombia
}

Recibido el 13 de abril de 2018; aceptado el 16 de octubre de 2018

Disponible en Internet el 20 de abril de 2019

\section{PALABRAS CLAVE}

Rehabilitación; Insuficiencia cardiaca crónica;

Calidad de vida;

Guía terapéutica

\begin{abstract}
Resumen
Introducción: Los programas de rehabilitación cardiaca integran la realización de ejercicio supervisado con prevención secundaria y soporte sicosocial que permite mejorar la adherencia a medidas con impacto claro sobre la morbilidad y mortalidad en pacientes con falla cardiaca. Metodología: Para el desarrollo de la guía de práctica clínica basada en la evidencia se siguieron los pasos definidos en la Guía Metodológica para la Elaboración de Guías de Práctica Clínica con Evaluación Económica en el Sistema General de Seguridad Social en Salud Colombiano del Ministerio de Salud y Protección Social de Colombia.

Resultados: Se establecen las recomendaciones para el desarrollo adecuado de un programa de rehabilitación cardiaca integral en pacientes con falla cardiaca, abordando aspectos de evaluación de capacidad funcional, efectividad y seguridad del ejercicio, dosificación, modalidad y rehabilitación en pacientes con dispositivos.
\end{abstract}

\footnotetext{
* Autor para correspondencia.

Correo electrónico: mmbt@une.net.co (J.M. Sénior).
} 
Conclusión: La práctica de ejercicio en pacientes con falla cardíaca estable ha demostrado disminuir las hospitalizaciones, mejorar la calidad de vida y la capacidad funcional y ser seguro en aquellos a quienes se les ha implantado un dispositivo.

(C) 2019 Sociedad Colombiana de Cardiología y Cirugía Cardiovascular. Publicado por Elsevier España, S.L.U. Este es un artículo Open Access bajo la licencia CC BY-NC-ND (http:// creativecommons.org/licenses/by-nc-nd/4.0/).

\section{KEYWORDS}

Rehabilitation; Chronic heart failure; Quality of life; Therapeutic guidelines
Clinical Practice Guidelines for the prevention, diagnosis, treatment, and rehabilitation of heart failure in a population greater than 18 years-old: B, C and D Classification. Cardiac rehabilitation component

\begin{abstract}
Introduction: Cardiac rehabilitation programs combine the performing of supervised exercise with secondary prevention and psycho-social support that helps to improve adherence to measures, with a clear impact on the morbidity and mortality in patients with heart failure.

Methodology: For the development of the evidence-based clinical practice guidelines, the steps followed were those defined in the Methodological Guidelines for the Preparation of Clinical Practice Guidelines with an Economic Assessment in the General Social Security System in Colombian Health by the Ministry of Health and Social Protection of Colombia.

Results: Recommendations were established for the adequate development of an integrated cardiac rehabilitation program for patients with heart failure. The aspects approached included the assessment of functional capacity, effectivity and safety of the exercise, dosification, methodology, and rehabilitation in patients with devices.

Conclusion: The practice of exercise in patients with stable heart has shown to reduce the number of hospital admissions, improve the quality of life and functional capacity. It has also shown to be safe in those that have a device implanted.

(C) 2019 Sociedad Colombiana de Cardiología y Cirugía Cardiovascular. Published by Elsevier España, S.L.U. This is an open access article under the CC BY-NC-ND license (http:// creativecommons.org/licenses/by-nc-nd/4.0/).
\end{abstract}

\section{Introducción}

Los programas de rehabilitación cardiaca integran la práctica de ejercicio supervisado con prevención secundaria y soporte sicosocial que permite mejorar la adherencia a medidas con claro impacto sobre morbilidad y mortalidad en pacientes con falla cardiaca (figs. 1 y 2$)^{1}$, y además optimizan la capacidad funcional y la calidad de vida e impactan desenlaces centrados en el paciente ${ }^{2}$, incluyendo aquellos con dispositivos de estimulación eléctrica o de soporte ventricular y los candidatos a trasplante de corazón ${ }^{3}$.

El ejercicio en sus diversas modalidades ha demostrado tener impacto en el riesgo de readmisión hospitalaria y mejoría en la calidad de vida, medida con diferentes herramientas; sin embargo, su efecto sobre la mortalidad se considera neutro, aunque algunos estudios demuestran reducción y generan bastante incertidumbre, dados los diseños y alternativas utilizadas ${ }^{4}$.

Es importante, por tanto, definir las estrategias adecuadas para recomendar en pacientes con falla cardiaca en los programas rehabilitación cardiaca, incluyendo las pruebas para evaluar la capacidad funcional, las modalidades de ejercicio con o sin la inclusión de entrenamiento con resistencia y fuerza, su intensidad y frecuencia ${ }^{5}$.

\section{Metodología}

Para el desarrollo de la guía de práctica clínica (GPC) basada en la evidencia se siguieron los pasos definidos en la Guía Metodológica para la Elaboración de Guías de Práctica Clínica con Evaluación Económica en el Sistema General de Seguridad Social en Salud Colombiano del Ministerio de Salud y Protección Social de Colombia (MSP) ${ }^{6}$. Se conformó el grupo desarrollador de la guía con participación de profesores, sociedades científicas y pacientes. Se elaboraron las preguntas con la estrategia PECOT, se definieron los desenlaces y su importancia y se hizo una búsqueda sistemática. Se efectuaron revisiones sistemáticas. La calidad de la evidencia de los estudios primarios en los ensayos clínicos controlados se hizo con base en los criterios definidos por Cochrane; para las revisiones sistemáticas y metaanálisis se utilizó el $\mathrm{AMSTAR}^{7}$ y para los estudios primarios de diagnóstico el QUADAS ${ }^{8}$. Los criterios para la evaluación de los estudios están referenciados en el trabajo del GRADE Working Group?.

Siguiendo el sistema GRADE, las recomendaciones se elaboraron de acuerdo con cinco aspectos: calidad de la evidencia, balance entre beneficios y riesgos, efectos adversos, relación costo-beneficio y valores y preferencias de los 


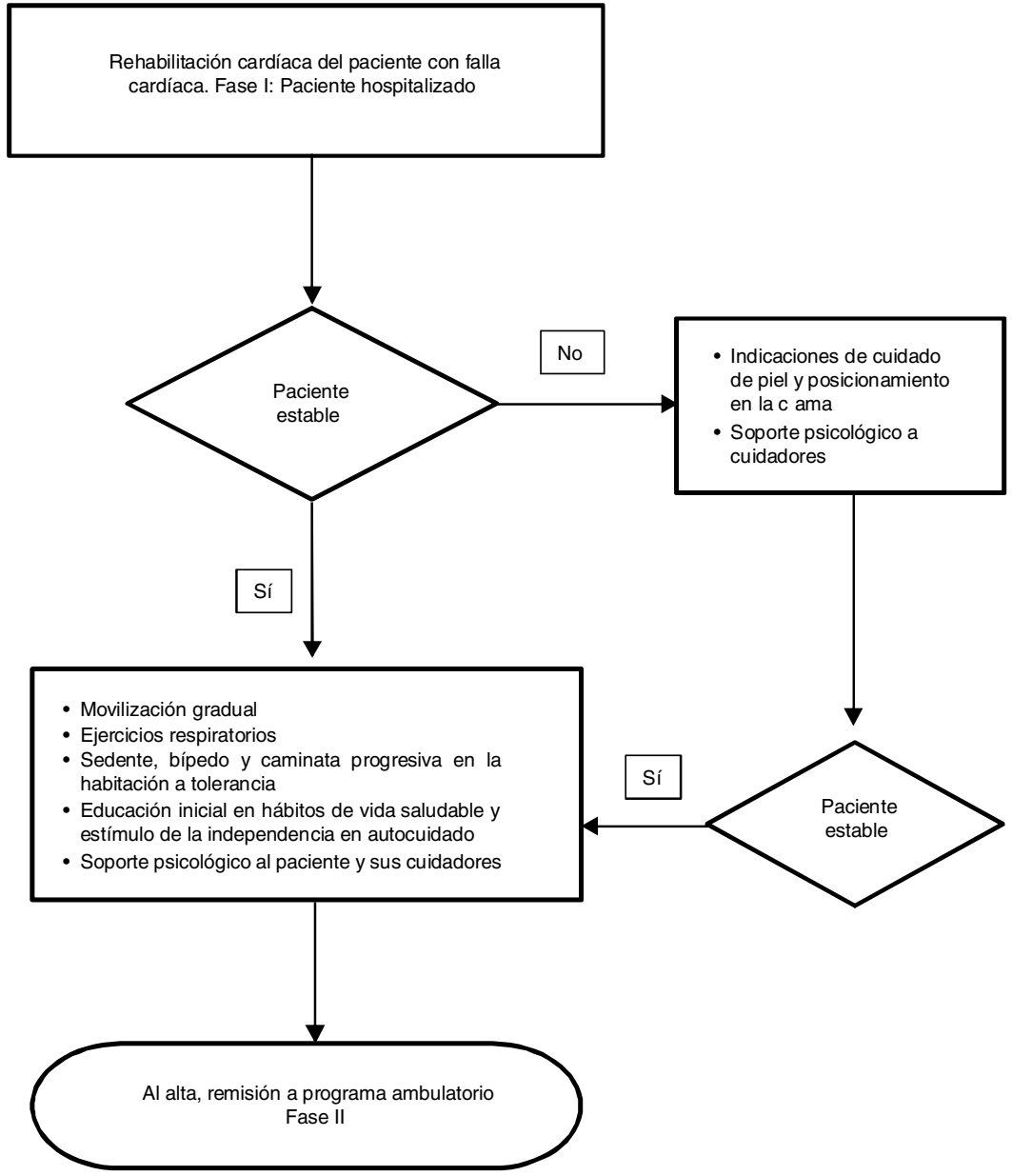

Figura 1 Rehabilitación cardíaca del paciente con falla cardíaca fase I: paciente hospitalizado Rehabilitación cardíaca del paciente con falla cardíaca Fase I: Paciente hospitalizado.

pacientes $^{10}$. Para la calidad de la evidencia se definieron cuatro niveles: muy baja, baja, moderada y alta, puntuando en forma individual cada desenlace, lo que genera recomendaciones fuertes o débiles, a favor de su utilización o en contra $^{11}$.

\section{Resultados}

\section{Prueba de esfuerzo cardiopulmonar vs. prueba de caminata de 6 minutos}

¿En pacientes con falla cardíaca la prueba de caminata de 6 minutos comparada con el consumo pico de oxígeno y el seguimiento a largo plazo, predicen las hospitalizaciones por todas las causas y la mortalidad por todas las causas 1 y 5 años después?
Recomendación Prueba de esfuerzo cardiopulmonar vs. prueba de caminata de 6 minutos

Fuerte a favor En pacientes con falla cardíaca sistólica con fracción de eyección $<40 \%$ y clase funcional NYHA II-IV, se recomienda la prueba de caminata de 6 minutos o prueba de esfuerzo cardiopulmonar para evaluar la capacidad física y predecir hospitalizaciones y muerte por todas las causas de acuerdo con las condiciones particulares de cada paciente. Calidad de la evidencia moderada $\oplus \oplus \oplus \mathrm{O}$

La capacidad funcional se define como la habilidad individual para efectuar un trabajo aeróbico con un consumo de oxígeno determinado y que permite realizar las diferentes actividades de la vida diaria ${ }^{12}$. La prueba de oro en la medición de la capacidad funcional en pacientes con falla 


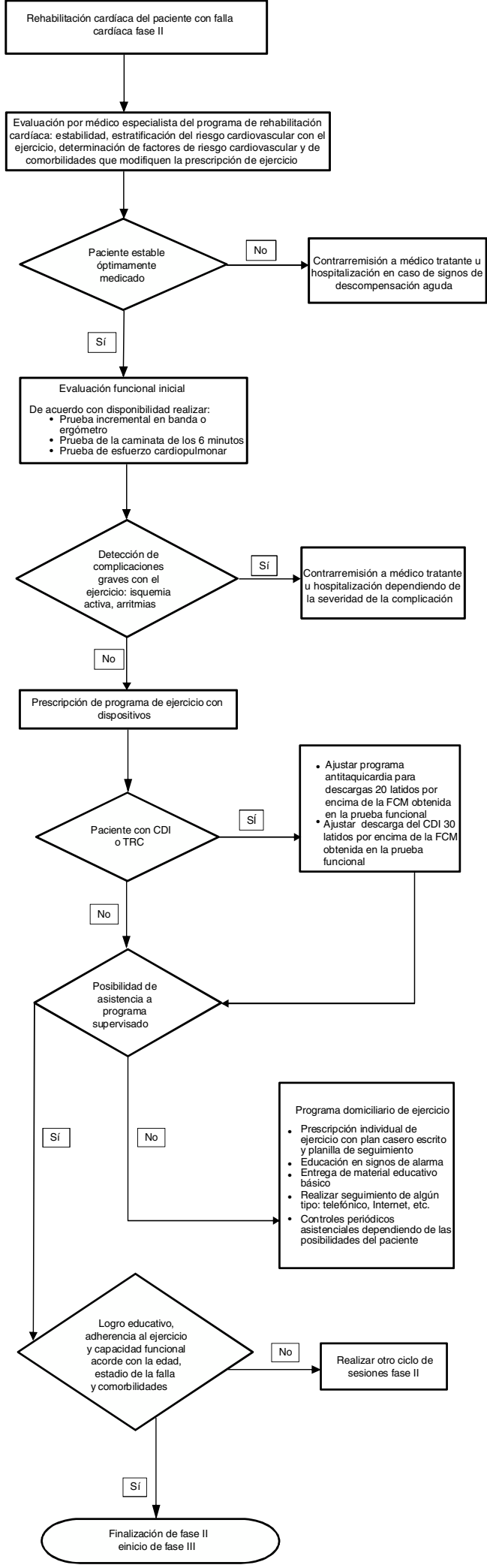

Figura 2 Rehabilitación cardíaca del paciente con falla cardíaca fase II. cardíaca es la prueba de esfuerzo cardiopulmonar; dentro de las mediciones realizadas en esta prueba, la determinación del consumo de oxígeno $\left(\mathrm{VO}_{2}\right.$ pico) ha demostrado ser el predictor más importante de pronóstico aunque también se han considerado otras mediciones ${ }^{13}$; sin embargo, por su mayor costo y poca disponibilidad se han desarrollado otras pruebas funcionales, dentro de las cuales la más utilizada es la prueba de la caminata de los 6 minutos.

\section{Prueba de esfuerzo vs. prueba de caminata de 6 minutos}

La revisión sistemática del año 2010 denominada "Examination of the Six Minute Walk Test to Determine Functional Capacity in People with Chronic Heart Failure: A Systematic Review" ${ }^{14}$, incluyó 14 estudios con un total de 933 pacientes con falla cardíaca; 11 estudios compararon la prueba de 6 minutos con la prueba cardiopulmonar ( 7 en banda y 4 ergométricas), 2 estudios con una prueba de esfuerzo convencional (2 ergométricas) y un estudio la comparó con un cuestionario de calidad de vida. Se observó moderada a buena correlación entre la prueba de seis minutos y el nivel pico de $\mathrm{VO}_{2}$ para predecir la capacidad funcional, dependiendo de la distancia caminada, con una exactitud entre el 83 al $91 \%$ si la distancia caminada era menor de 450 a 490 metros, respectivamente. Se observó una correlación más fuerte en pacientes con falla cardíaca avanzada con distancias caminadas menores a 300 metros o con un bajo consumo pico de oxígeno $(<10 \mathrm{ml} / \mathrm{kg} / \mathrm{min})$.

\section{Discusión}

La prueba de seis minutos se puede reproducir en el grupo de pacientes con falla cardíaca (NYHA I-II) y se correlaciona moderadamente con la prueba cardiopulmonar. Por tanto, se presenta como una herramienta de evaluación fidedigna y constituye una alternativa adecuada, segura y de bajo costo para la prescripción de ejercicio físico aeróbico en pacientes con falla cardíaca con clase funcional no avanzada.

\section{Efectividad y seguridad del ejercicio en pacientes con falla cardíaca estable}

1 Efectividad y seguridad del ejercicio en pacientes con falla cardiaca estable ¿En pacientes con falla cardíaca estable cuál es la efectividad y seguridad del ejercicio comparado con no hacerlo para mejorar la capacidad funcional, la calidad de vida, disminuir las rehospitalizaciones, los episodios de descompensación agudos, la mortalidad, evitar el edema pulmonar agudo, el episodio coronario agudo o las arritmias malignas a dos y seis meses? 
Recomendación priorizada

Ejercicio regular supervisado para disminuir hospitalización

Fuerte a favor Se recomienda el ejercicio aeróbico regular y supervisado en pacientes con falla cardíaca estables, con fracción de eyección disminuida y clase funcional NYHA II a III para reducir el riesgo de hospitalizaciones por falla cardíaca.

Calidad de la evidencia moderada $\oplus \oplus \oplus \mathrm{O}$

Recomendación Ejercicio regular supervisado para mejorar priorizada calidad de vida

Fuerte a favor

Se recomienda el ejercicio aeróbico regular y supervisado en pacientes con falla cardíaca estables, con fracción de eyección disminuida y clase funcional NYHA II a III para mejorar la calidad de vida por falla cardíaca.

Calidad de la evidencia baja $\oplus \oplus \oplus \mathbf{O}$

Recomendación Ejercicio regular supervisado en falla priorizada cardíaca con fracción de eyección preservada

Débil a favor Se sugiere el ejercicio aeróbico regular y supervisado en pacientes con falla cardíaca estables, con fracción de eyección preservada y clase funcional NYHA II a III para mejorar la calidad de vida y la capacidad funcional.

Calidad de la evidencia baja $\oplus \oplus \oplus \mathrm{O}$

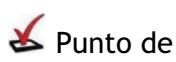
buena práctica

El programa de rehabilitación cardíaca debe ser liderado por especialista en Cardiología y/o Medicina deportiva y/o fisiatra y/o internista con entrenamiento en rehabilitación cardíaca, con disponibilidad médica durante las sesiones de entrenamiento.

Debe vincular enfermera y fisioterapeuta con entrenamiento en rehabilitación cardíaca.

Debe haber disponibilidad de cardiólogo, sicólogo, terapeuta ocupacional y nutricionista.

Los componentes básicos son: evaluación clínica del paciente, electrocardiograma en reposo, prueba de esfuerzo para evaluar capacidad funcional, prueba de caminata de 6 minutos, prueba para tamizaje de depresión, escala para evaluar calidad de vida, prescripción del ejercicio, educación y consejería.

Taylor et al. en una revisión sistemática liderada por el grupo de Colaboración Cochrane actualizada en $2014^{15}$, identificaron 33 estudios con rehabilitación basada en ejercicio para un total de 4740 pacientes, predominantemente con falla cardíaca con fracción de eyección reducida y clase II y III de la NYHA; todos los estudios evaluaron intervención aeróbica y 11 incluyeron ejercicios de resistencia; 5 de los estudios evaluaron intervenciones de ejercicio realizadas en casa. No se encontró evidencia para reducción de la mortalidad estadísticamente significativa. Respecto a las admisiones hospitalarias se halló evidencia en 15 de los artículos de reducción en el número de hospitalizaciones en el grupo de ejercicio comparado con el grupo de control hasta los 12 meses de seguimiento (RR 0.75 IC 95\% $0.62-0.92, \mathrm{I}^{2}=0 \%$ ); específicamente por admisiones relacionadas con falla cardíaca se encontró evidencia en 12 de los estudios (RR 0.61 IC 95\% 0.46-0.80, $I^{2}=34 \%$ ). En cuanto a las admisiones hospitalarias por todas las causas en seguimiento superior de los 12 meses se encontró evidencia en 5 de los estudios y no hubo diferencia estadísticamente significativa (RR 0.92 IC 95\% 0.66-1.29, I²=63\%).

Para el desenlace de calidad de vida relacionada con salud, 19 de los 33 estudios incluidos utilizaron una medida validada de HRQoL (Health Related Quality of Life) y reportaron mejoría en el grupo intervenido, aunque con heterogeneidad estadística.

\section{Discusión}

A pesar de la baja calidad de los estudios analizados, 33 ECC analizados concuerdan en que el ejercicio provee beneficios para mejorar la calidad de vida relacionada con la salud y reduce la hospitalización por falla cardíaca sistólica. No se halló evidencia que sustentara que los programas de ejercicio de entrenamiento incrementen el riesgo de muerte en menos de un año, y por el contrario, se encontró tendencia a la reducción de la mortalidad con estudios de seguimiento a 12 meses. Los eventos adversos importantes no fueron mayores en el grupo de cuidado usual comparados con el ejercicio. Sin embargo, es importante tener en cuenta que en la mayoría de los estudios incluidos en las revisiones sistemáticas analizadas, el ejercicio se hizo de manera supervisada, por lo que la seguridad depende de que la actividad física se realice de esta manera (fig. 3).

\section{Dosificación del ejercicio en pacientes con falla cardíaca estable}

¿En pacientes con falla cardíaca el ejercicio con intervalos comparado con el continuo, el ejercicio de intensidad leve comparada con la moderada o alta, o el ejercicio aeróbico más ejercicios de fuerza comparado con el aeróbico solo, mejora la capacidad funcional, la calidad de vida y la adherencia, disminuye las re-hospitalizaciones y la mortalidad, y evita la descompensación aguda y las arritmias malignas, dos y seis meses después de iniciar el programa de rehabilitación cardiaca? 


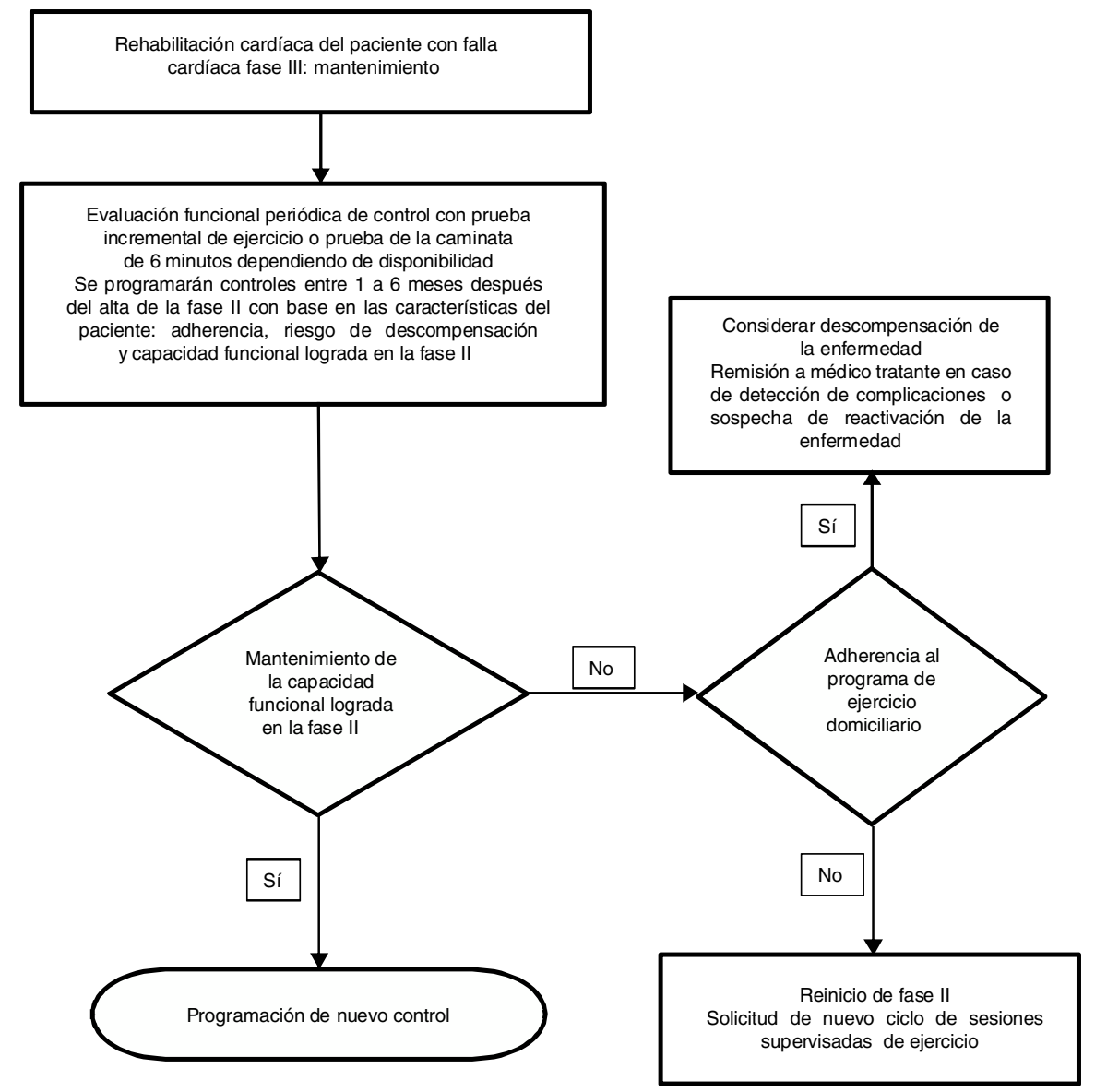

Figura 3 Rehabilitación cardíaca del paciente con falla cardíaca fase III: mantenimiento.

Recomendación Ejercicio aeróbico supervisado a intervalos Débil a favor Se sugiere realizar ejercicio aeróbico a intervalos supervisado, en pacientes con falla cardíaca que lo toleren, para mejorar la capacidad funcional y la calidad de vida. Calidad de la evidencia baja $\oplus \oplus$ OO

Recomendación Ejercicio aeróbico supervisado a intensidad moderada a vigorosa

Fuerte a favor Se recomienda realizar ejercicio aeróbico supervisado a intensidad moderada y vigorosa, de acuerdo con la tolerancia, en pacientes con falla cardíaca para mejorar la capacidad funcional, sin aumentar el riesgo de muerte, hospitalizaciones, ni disminución de la adherencia.

Calidad de la evidencia baja $\oplus \oplus$ Oo

Recomendación Ejercicio aeróbico combinado con fuerza Débil a favor Se sugiere realizar ejercicio aeróbico combinado con fuerza para mejorar la capacidad funcional.

Calidad de la evidencia baja $\oplus \oplus$ oo

Las dos modalidades de ejercicio más utilizadas en el paciente con falla cardíaca son el aeróbico y el de fuerza o resistencia muscular. En la actualidad, el modelo aceptado en la clínica para la prescripción del ejercicio es el entrenamiento aeróbico continuo de intensidad moderada ${ }^{16}$.

La modalidad aeróbica es la más utilizada porque ha demostrado mejoría de la capacidad funcional y disminución de la morbimortalidad. El ejercicio aeróbico puede hacerse en diferentes intensidades (tabla 1) y de manera continua o a intervalos. En la modalidad aeróbica continua se realiza una fase inicial de calentamiento de 5 a 10 minutos de baja intensidad y luego se eleva la intensidad hasta una meseta (fase de acondicionamiento), que se mantiene hasta los últimos 5 a 10 minutos cuando la intensidad se vuelve a disminuir (fase de enfriamiento). La intensidad del ejercicio aeróbico se puede medir a través de varios métodos: el de la frecuencia cardíaca máxima (FCM), el de la frecuencia cardíaca de reserva (FCR), el del pico máximo de oxígeno $\left(\% \mathrm{VO}_{2}\right.$ máximo), y por medición de equivalentes metabólicos (MET) o por escalas de percepción subjetiva de la intensidad del ejercicio.

En la modalidad aeróbica a intervalos también están las fases de calentamiento y enfriamiento, pero la fase de acondicionamiento se hace con episodios cortos alternos (10 a 30 segundos) de alta a moderada intensidad (50 al 100\% del $\left.\mathrm{VO}_{2 \text { máx }}\right)$, intercalados con episodios de baja intensidad más largos (60 a 80 segundos) que no superen el umbral anaeróbico. 
Tabla 1 Intensidad de ejercicio aeróbico

\begin{tabular}{|c|c|c|c|c|c|}
\hline \multirow[t]{2}{*}{ Intensidad } & \multicolumn{4}{|c|}{ Ejercicio aeróbico } & \multirow{2}{*}{$\begin{array}{l}\text { Ejercicio de fuerza } \\
\% 1 \mathrm{RM}\end{array}$} \\
\hline & $\%$ FCR & $\% \mathrm{FCM}$ & $\% \mathrm{VO}_{2 \max }$ & MET & \\
\hline Muy suave & Menos 30 & Menos 57 & Menos 37 & Menos 2 & Menos 30 \\
\hline Suave & $30-39$ & $57-63$ & $37-45$ & $2-2.9$ & $30-49$ \\
\hline Moderada & $40-59$ & $64-76$ & $46-63$ & $3-5.9$ & $50-69$ \\
\hline Vigorosa & $60-89$ & $77-95$ & $64-90$ & $6-8.7$ & $70-84$ \\
\hline Alta & Mayor o igual a 90 & Mayor o igual 96 & Mayor o igual 91 & Mayor o igual 8.8 & Mayor o igual 85 \\
\hline
\end{tabular}

Clasificación de la intensidad del ejercicio aeróbico y de resistencia según la intensidad. Tomado de: American College of Sports Medicine ${ }^{17}$. FRC: frecuencia cardíaca de reserva, $\mathrm{FCM}$ : frecuencia cardíaca máxima, $\mathrm{VO}_{2 \text { max }}$ : pico máximo de oxígeno, MET: unidad de equivalente metabólico.

\section{Ejercicio aeróbico a intervalos vs. ejercicio aeróbico continuo}

Smart et al. ${ }^{18}$ publicaron una revisión sistemática y un metaanálisis de 13 ensayos clínicos con 446 pacientes de ejercicios combinados de fuerza con ejercicio aeróbico intermitente y ejercicio continuo en pacientes con falla cardíaca. Los resultados encontrados fueron estadísticamente significativos a favor del ejercicio intermitente cuando se comparó con controles sedentarios (diferencia de medias $\mathrm{VO}_{2}$ máximo $1.58 \mathrm{ml} / \mathrm{kg} / \mathrm{min}$ IC $95 \% 1.13-2.04$ ), y cuando se comparó con el ejercicio continuo (diferencia de medias $\mathrm{VO}_{2} 1.04 \mathrm{ml} / \mathrm{kg} / \mathrm{min}$ IC 95\% 0.42-1.66), ambos resultados con alta heterogeneidad. En cuanto al cambio del VE $/ \mathrm{VCO}_{2}$, los resultados estuvieron también a favor del ejercicio intermitente cuando se comparó con el grupo de controles sedentarios (diferencia de medias - 1.50 IC 95\% -2.64-0.37) y con el grupo de ejercicio continuo (diferencia de medias -1.35 IC 95\% - 2.15- -0.55). Tras el análisis se concluyó que el ejercicio intermitente produce mayores beneficios en el pico $\mathrm{VO}_{2}$ y $\mathrm{VE} / \mathrm{VCO}_{2}$.

\section{Ejercicio aeróbico de intensidad leve comparado con intensidad moderada, vigorosa o alta}

El metaanálisis de Ismail $^{19}$, incluyó 74 estudios con las siguientes intervenciones: 9 (11.8\%) de alta intensidad, 38 (50\%) de intensidad vigorosa, 24 (31.6\%) de intensidad moderada y 5 (6.6\%) de baja intensidad, para un total de 3.265 pacientes en el grupo de intervención y 2.612 en el grupo control. Los resultados demuestran mejoría del $23 \%$ respecto al control en el consumo pico de oxígeno $\left(\mathrm{VO}_{2}\right.$ diferencia de medias $3.33 \mathrm{ml} / \mathrm{kg} / \mathrm{min}$ IC $95 \%$ 0.53-6.13 ml) con entrenamiento de alta intensidad en comparación con el grupo de control; en cuanto a intensidad vigorosa $8 \%$ de cambio respecto al control (diferencia de media $2.27 \mathrm{ml} / \mathrm{kg} / \mathrm{min}$ IC 95\%1.70-2.84 ml). En intensidad moderada la diferencia de medias fue de $2.17 \mathrm{ml} / \mathrm{kg} / \mathrm{min}$ (IC 95\% 1.34-2.99 ml) y en lo referente a baja intensidad la diferencia de medias fue apenas de $1.04 \mathrm{ml} \mathrm{ml} / \mathrm{kg} / \mathrm{min}$ (IC 95\% de $-2.50-4.57 \mathrm{ml}$ ); esta última no estadísticamente significativa. Los autores del artículo concluyen que a mayor intensidad del ejercicio, mejor capacidad cardiorrespiratoria.

\section{Ejercicio aeróbico solo comparado con el ejercicio aeróbico combinado con ejercicios de fuerza}

El metaanálisis de Smart $(2013)^{20}$ concluye que al comparar ejercicio intermitente solo versus ejercicio intermitente combinado con ejercicio de fuerza, el resultado estuvo a favor del ejercicio combinado (diferencia en la media pico de $\mathrm{VO}_{2}-1.10 \mathrm{ml} / \mathrm{kg} / \mathrm{min}$ IC $\left.95 \%-1.83-0.37\right)$.

\section{Discusión}

En cuanto a la dosificación del ejercicio aeróbico en falla cardíaca los estudios concuerdan en determinar que la intensidad del entrenamiento y su realización a intervalos tienen una relación directa con la mejoría de la capacidad funcional, sin evidencia de efectos adversos y sin disminuir la adherencia, pero para lograr esta seguridad su práctica debe ser supervisada y en centros hospitalarios.

\section{Ejercicio protocolizado en pacientes con terapia de resincronización cardiaca, cardiodesfibrilador implantable y terapia de resincronización cardiaca-cardiodesfibrilador implantable}

¿En pacientes con falla cardíaca con dispositivos de terapia de resincronización cardiaca, cardiodesfibrilador implantable y terapia de resincronización cardiacacardiodesfibrilador implantable el ejercicio protocolizado comparado con el manejo estándar o no hacerlo, mejora la capacidad funcional, la calidad de vida y la adherencia, disminuye las rehospitalizaciones y la mortalidad, y evita la descompensación aguda y las arritmias malignas a dos y seis meses después de iniciar el programa de rehabilitación cardiaca? 


\begin{tabular}{ll}
\hline Recomendación & Ejercicio protocolizado en pacientes con \\
& terapia de resincronización cardiaca, \\
& cardiodesfibrilador implantable y terapia de \\
& resincronización cardiaca-cardiodesfibrilador \\
& implantable \\
Fuerte a & Se recomienda realizar ejercicio supervisado \\
favor & para mejorar la capacidad funcional y la \\
& calidad de vida en pacientes con falla \\
cardíaca estables con cardiodesfibrilador \\
implantable y/o terapia de resincronización \\
cardíaca, sin que esto aumente el riesgo de \\
descargas, mortalidad o de eventos adversos. \\
Calidad de la evidencia muy baja $\oplus$ ooo para \\
mejoría de capacidad funcional y calidad de \\
vida \\
Calidad de la evidencia moderada $\oplus \oplus \oplus$ o \\
para seguridad del ejercicio en pacientes con \\
terapia de resincronización cardiaca y \\
cardiodesfibrilador implantable
\end{tabular}

La realización de ejercicio en pacientes con falla cardíaca estable ha demostrado disminuir las hospitalizaciones, y mejorar la calidad de vida y la capacidad funcional en pacientes con falla cardíaca. El subgrupo de pacientes con terapia de resincronización cardiaca y cardiodesfibrilador implantable fue incluido en los estudios que avalaron esta recomendación; sin embargo, se tienen dudas con relación a la seguridad del ejercicio en este tipo de pacientes y la posibilidad de complicaciones específicas tales como la presencia de descargas inadecuadas.

En el ensayo clínico aleatorizado de Patwala et al. ${ }^{21}$ que incluyó 50 pacientes con falla cardiaca estado funcional NYHA III-IV, se demostró que tres meses después de la terapia de resincronización cardiaca hubo mejoría en el $\mathrm{VO}_{2}$ pico pasando de $16.12+-3.44 \mathrm{ml} / \mathrm{min} / \mathrm{kg}$ a $18.41 \pm 3.56 \mathrm{ml} / \mathrm{min} / \mathrm{kg}(\mathrm{p}<0.001)$; en cuanto a calidad de vida hubo un cambio en el MLWHF de $61.8 \pm 17.7$ a $31.8 \pm 19.5$ tras 3 meses y para torque músculo esquelético pico derecho $135.0 \pm 50.3$ vs. $144.8 \pm 57.6(p<0.009)$; izquierdo $135.0+-$ 50.4 vs. $143.6 \pm 53.1(\mathrm{p}<0.004)$.

El estudio clínico aleatorizado de Conraads et al. ${ }^{22}$ incluyó 17 pacientes derivados a terapia de resincronización cardiaca, con tratamiento farmacológico estable más de 1 mes, que fueron aleatorizados a dos grupos: terapia farmacológica con entrenamiento de resistencia (8 pacientes), y demostró aumento del $\mathrm{VO}_{2}$ pico $5.5 \mathrm{ml} / \mathrm{kg} / \mathrm{min}(+40 \%$ contra $+16 \%, p=0.005)$, Wattmax $(+43 \%$ contra $+13 \% p=0.0005)$ y poder circulatorio $(+74 \%$ contra $+32 \% p=0.01)$, el cual fue significativamente mayor en el grupo de entrenamiento de resistencia comparado con pacientes con terapia de resincronización cardiaca sin entrenamiento.

El estudio clínico aleatorio del grupo de Belardinelli $i^{23}$ incluyó 52 hombres, en clase funcional II y III de la NYHA, con miocardiopatía isquémica quienes tenían cardiodesfibrilador implantable al menos por tres meses con o sin terapia de resincronización cardiaca, aleatorizados a dos grupos: el $\mathrm{T}$ en un programa de ejercicio supervisado a
$60 \%$ del consumo pico y el $\mathrm{C}$ sedentario. A las 8 semanas solo los pacientes en el grupo de ejercicio habían mejorado el consumo pico de oxígeno; en cuanto a la calidad de vida mejoró significativamente en pacientes con cardiodesfibrilador implantable -terapia de resincronización cardiaca ( $54 \pm 18$ a $40 \pm 20 ; p<0.0001$ comparado con $C y<0.02$ comparado con cardiodesfibrilador implantable). Los subgrupos de ejercicio con cardioesfibrilador implantable o cardiodesfibrilador implantable-terapia de resincronización cardiaca, no tuvieron efectos adversos.

El estudio COPE-ICD de Berg et al. ${ }^{24}$, incluyó 196 pacientes al grupo control o al grupo de intervención, más un componente psicosocial de 1 año de duración realizado por 2 enfermeras y un componente de ejercicio individualizado con énfasis en fuerza y resistencia realizado por 2 fisioterapeutas. Los resultados mostraron que el SF-36 en su componente físico (PCS) fue de 42.6 y el componente mental (MCS) fue de 47.1. En cuanto a la capacidad de caminar media (6 MWT) fue de $417 \mathrm{~m}$. Los autores concluyen que un programa de rehabilitación integral mejora la calidad de vida y la función física.

El estudio COPE-ICD de Berg et al. ${ }^{25}$ comparó el programa de rehabilitación integral con cuidado usual y demostró mejoría del consumo de oxígeno $23.0 \mathrm{ml} / \mathrm{min} / \mathrm{kg}$ (IC 95\% 20.9-22.7) comparado con $20.8 \mathrm{ml} / \mathrm{min} / \mathrm{kg}$ (IC $95 \% 18.9$ $22.7 \mathrm{ml} / \mathrm{min} / \mathrm{kg})$ en el grupo control $(\mathrm{p}=0.004)$. Igualmente, hubo mejoría en el estado de salud medido por SF-36 media 62.8 (IC 95\% 58.1-67.5) comparado con 64.4 (IC 95\% 59.6-69.2); a los 6 meses 66.7 (IC 95\% 61.5-72.0) comparado con 61.9 (IC 95\% 56.1-67.7); a los 12 meses 63.5 (IC 95\% 57.7-69.3) comparado con 62.1 (IC 95\% 56.2-68.0); de acuerdo con los análisis se encontró una diferencia significativa a favor del grupo de tratamiento. En cuanto al número de descargas el grupo de intervención tuvo 0.20 (IC 95\% 0.02-0.38) comparado con 0.43 (IC 95\% 0.03-0.84, $\mathrm{p}=0.76$ ), sin diferencias significativas entre estos.

\section{Discusión}

La práctica de ejercicio aeróbico regular y supervisado en pacientes con falla cardíaca estable con fracción de eyección disminuida, clase funcional II a III de la NYHA, a quienes se les haya implantado terapia de resincronización cardiaca o cardiodesfibrilador, disminuye el riesgo de hospitalizaciones por falla cardíaca y mejora la calidad de vida y capacidad funcional y no se aumentan los eventos adversos ni las complicaciones.

\section{Ejercicio supervisado vs. ejercicio en casa}

¿En pacientes con falla cardíaca el ejercicio supervisado vs. el ejercicio en casa mejora la capacidad funcional, la calidad de vida y la adherencia, disminuye rehospitalizaciones y mortalidad, evita la descompensación aguda y las arritmias malignas a dos y seis meses después de iniciar el programa de rehabilitación cardiaca? 


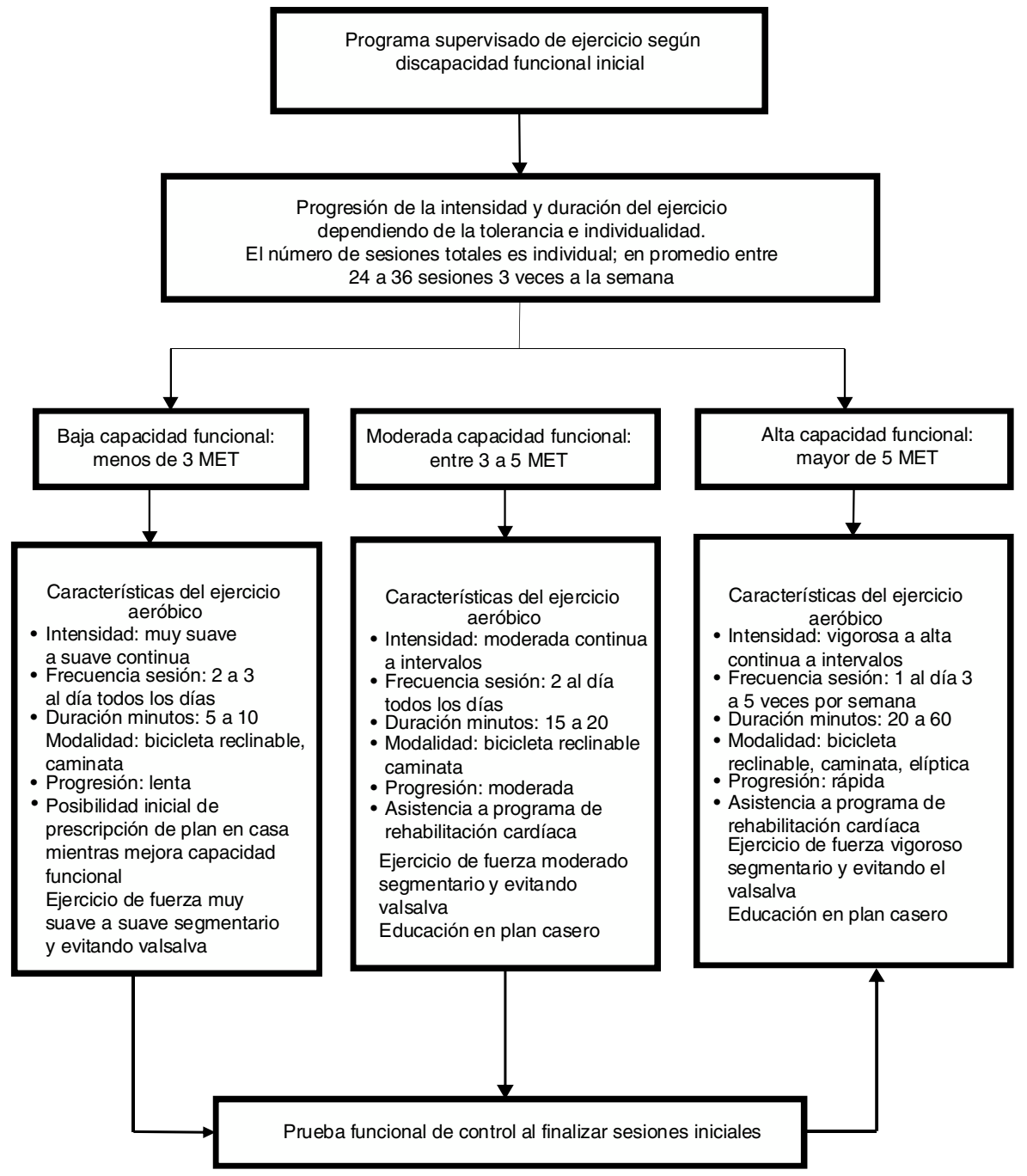

Figura 4 Programa supervisado de ejercicio según capacidad funcional inicial.

Recomendación

Débil a favor

Punto de buena práctica

\section{Ejercicio en casa}

Se sugiere realizar ejercicio en casa en pacientes con falla cardíaca, para mejorar la capacidad funcional, cuando no tengan posibilidad de asistir a un centro de ejercicio supervisado.

Calidad de la evidencia muy baja $\oplus$ OO

El programa en casa incluye estratificación inicial del riesgo con el ejercicio, prescripción personal y seguimiento periódico telefónico o personal.

Los programas de ejercicio de rehabilitación cardíaca en los pacientes con falla cardíaca pueden hacerse de manera supervisada en centros hospitalarios o de manera ambulatoria con indicaciones dadas por educación personal o audiovisual. Se han creado programas de ejercicio en casa, en los cuales el paciente recibe instrucción de manera personal o mediante educación audiovisual (material escrito, videos) y seguimiento telefónico o por Internet para garantizar la adherencia y detectar signos de alarma con el ejercicio (fig. 4).
El estudio de Cowie et al. ${ }^{26}$ incluyó 60 pacientes con falla cardíaca estable, a quienes se les optimizó el tratamiento farmacológico; fueron aleatorizados a un grupo supervisado que asistió a la sesión de ejercicio en el centro de rehabilitación dirigida por una fisioterapeuta y al control, al cual se le dio un DVD y una cartilla y se le hizo seguimiento telefónico a cargo de una fisioterapeuta cada dos semanas.

Los resultados mostraron mejoría en la capacidad del ejercicio en ambos grupos hospitalarios $(+71 \mathrm{~m}, p 0.01)$ y en casa (+44 $\mathrm{m}, p$ 0.02) después de 8 semanas, siendo estadísticamente significativos comparado con el grupo control $(p$ 0.01 hospitalario y $p 0.03$ casa), pero sin diferencia estadística entre los dos grupos de ejercicio al final del programa $(p$ 0.47 ). Tampoco se encontraron diferencias significativas en el MHLFQ ni en el SF-36 (componente físico); sin embargo, el componente mental SF-36 fue mejor en el grupo hospitalario comparado con el grupo en casa ( $p$ 0.04).

El estudio clínico con asignación aleatoria de Karapolat et al. ${ }^{27}$ incluyó 74 pacientes distribuidos en grupo de entrenamiento hospitalario o en casa; los resultados mostraron que ambos programas de ejercicio mejoran el $\mathrm{VO}_{2}$ pico, la caminata de 6 minutos, el SF-36, BDI y la fracción 
de eyección del ventrículo izquierdo sin mostrar diferencia estadísticamente significativa entre los dos grupos, sin presencia de eventos adversos en cada uno de esos grupos y con porcentajes de adherencia del $90 \%$ para el grupo hospitalario y del $87.5 \%$ en el grupo de ejercicio en casa.

Por su parte, el estudio de Daskapan et al. ${ }^{28}$ incluyó 22 pacientes con falla cardíaca crónica estable, asignados de manera aleatoria a los grupos de ejercicio hospitalario o en casa. El grupo en casa recibió una sesión inicial de entrenamiento y educación en medición del pulso y realización de la actividad con Borg entre 11 a 15; con seguimiento telefónico semanal. Los resultados mostraron que la duración del ejercicio incrementó significativamente en los grupos de ejercicio hospitalario y en casa $(\mathrm{p}<0.05)$; en cuanto al grupo hospitalario hubo una mejoría notable en el pico $\mathrm{VO}_{2}$ con ejercicio supervisado; sin embargo, en el grupo de ejercicio en casa no hubo cambio significativo en el $\mathrm{VO}_{2}$ pico.

\section{Discusión}

El ejercicio mejora la capacidad funcional y los programas deben ser formulados de acuerdo con la capacidad funcional de cada paciente. El ejercicio en casa puede ser una alternativa para los pacientes que no deseen participar en un programa supervisado o tengan dificultades en el acceso; no obstante, su implementación en el sistema de salud no es fácil, dado que en el manual único de procedimientos no están incluidas las actividades necesarias para su seguimiento, tales como contacto telefónico, vía Internet y visitas domiciliarias.

\section{Programa de rehabilitación cardíaca de ejercicio vs. programa integral}

¿En pacientes con falla cardíaca un programa de rehabilitación cardíaca basado en el ejercicio comparado con un programa integral (educación, intervención psicológica y cuidados ergonómicos) mejora la capacidad funcional, la calidad de vida y la adherencia, disminuye rehospitalizaciones y mortalidad, y evita la descompensación aguda y las arritmias malignas a dos y seis meses después de iniciar el programa de rehabilitación cardiaca?

\section{Recomendación Programa integral}

Fuerte a favor En pacientes con falla cardíaca se recomienda realizar ejercicio como parte de un programa integral en lugar de solo ejercicio cuando sea posible contar con apoyo integral (educación e intervenciones psicosociales) para mejorar la calidad de vida y las hospitalizaciones.

Calidad de la evidencia muy baja $\oplus$ ooo

Los programas integrales de rehabilitación cardíaca involucran educación, apoyo psicológico e indicaciones de reintegro funcional y social. Estas actividades son dirigidas por los integrantes del programa multidisciplinario ya sea médico, psicólogo, nutricionista, terapeuta físico, terapeuta ocupacional o enfermera. Sin embargo, no está claro si estos programas integrales son más efectivos que la sola realización de ejercicio.
Tras la estrategia de búsqueda utilizada no se encontraron artículos que compararan ejercicio con un programa de rehabilitación cardíaca integral, por lo cual se extrapoló información de manera indirecta de los artículos encontrados en el metaanálisis de Taylor et al. ${ }^{15}$, titulado "Exercise-based rehabilitation for heart failure (Review)"', donde se describe que fueron seleccionados 12 artículos de rehabilitación integral que incluían un programa de ejercicio, sumado a intervenciones psicosociales y educacionales, comparándolo con cuidado usual o ejercicio en casa. En esta revisión se hizo una metarregresión para determinar si la realización de ejercicio como parte de un programa integral tenía desenlaces diferentes a los de los programas de solo ejercicio y se encontró que no había diferencia entre ellos respecto a todas las causas de mortalidad ( $p 0.39)$ y hospitalización ( $p$ 0.26) y calidad de vida medidos con el cuestionario de Minnesota ( $p$ 0.42) y con todas las otras escalas ( $p$ 0.82).

Davidson et al. ${ }^{29}$ realizaron un estudio clínico aleatorio con 105 pacientes (53 ejercicio y 52 controles), con falla cardíaca de cualquier etiología, clase funcional I-IV de la NYHA. La intervención fue un programa de ejercicio sumado a un componente individualizado de ejercicio adaptado a la capacidad funcional y las circunstancias sociales de cada paciente; en el programa se incluyó personal de enfermería, farmacia, fisioterapia, terapia ocupacional y nutrición en sesiones grupales. Aunque el estudio fue suspendido en forma prematura, los resultados mostraron que el grupo de intervención tuvo menos admisiones hospitalarias por cualquier causa 44 vs. 69\% (OR 0.20 IC 95\% 0.07-0.58, p 0.01), y por eventos coronarios mayores agudos 24 vs. 55\% (OR 0.12 IC 95\% 0.04-0.37, p 0.001); los participantes en el grupo de intervención tenían más probabilidades de estar vivos a los 12 meses 93 vs. 79\% (OR 3.85 IC 95\% 1.03-14.42, p 0.03). Los puntajes de calidad de vida mejoraron a los 3 meses comparados con la línea de base, intervención ( $\mathrm{t}-4.37 p<0.0001$ control $t-3.52 p<0.01)$. También hubo mejoría en la prueba de caminata de 6 minutos a los 3 meses comparado con las características basales en el grupo de intervención (t 3.40; p 0.01).

Por lo anterior, el grupo encargado de la guía desarrolló un nuevo metaanálisis que incluyó 7 estudios con 462 pacientes, en el cual no se demostraron diferencias significativas en mortalidad (RR 0.65 IC 95\% 0.34-1.21) ni en hospitalizaciones (RR 0.74 IC 95\% 0.54-1.02), pero sí en calidad de vida [diferencia de medias $-0.31(-0.55-0-07)$ ], aunque sin importancia clínica.

\section{Discusión}

La participación en un programa de ejercicio como parte de un programa integral no genera mayores riesgos comparado con no hacerlo. Una de las dificultades del análisis de estos estudios es la definición de integralidad, que para muchos de ellos es hacer solo una actividad, generalmente educativa, además del ejercicio. Desde el punto de vista de la rehabilitación, el concepto de integralidad requiere una visión y un apoyo psicológico y social individualizado en el cual se efectúen actividades, no solo educativas. 


\section{Entidades participantes}

Universidad de Antioquia, Grupo para el Estudio de las Enfermedades Cardiovasculares y Grupo de Rehabilitación en Salud, en asocio con Alianza CINETS: Universidad Pontificia Javeriana y Universidad Nacional de Colombia; y las siguientes asociaciones científicas: Asociación Colombiana de Medicina Interna -ACMI-, Sociedad Colombiana de Cardiología y Cirugía Cardiovascular, American College of Physicians -ACP- Capítulo Colombia, Sociedad Latinoamericana de Medicina Interna -SOLAMI-, International Society of Internal Medicine -ISIM-.

\section{Financiación}

El desarrollo de esta guía fue financiado por el Ministerio de Salud y Protección Social, por medio de la convocatoria 637-2013 del Departamento Administrativo de Ciencia, Tecnología e Innovación (COLCIENCIAS), mediante Código 111563738927 con la Universidad de Antioquia. Contrato número 773 de 2013.

\section{Conflicto de intereses}

Ninguno.

\section{Bibliografía}

1. Ades PA, Keteyian SJ, Balady GJ, Houston-Miller N, Kitzman DW, Mancini DM, et al. Cardiac rehabilitation exercise and selfcare for chronic heart Failure. JACC Heart Fail. 2013;1:540-7, http://dx.doi.org/10.1016/j.jchf.2013.09.002./.

2. Keteyian SJ, Squires RW, Ades PA, Thomas RJ. Incorporating patients with chronic heart failure into outpatient cardiac rehabilitation: practical recommendations for exercise and self-care counseling-a clinical review. J Cardiopulm Rehabil Prev. 2014;34:223-32, http://dx.doi.org/10. 1097/HCR. 0000000000000073./.

3. Forman DE, Sanderson BK, Josephson RA, Raikhelkar J, Bittner V. American College of Cardiology's Prevention of Cardiovascular Disease Section Heart Failure as a Newly Approved Diagnosis for Cardiac Rehabilitation: Challenges and Opportunities. J Am Coll Cardiol. 2015;65:2652-9, http://dx.doi.org/ 10.1016/j.jacc.2015.04.052./.

4. Taylor RS, Sagar VA, Davies EJ, Briscoe S, Coats AJ, Dalal H, et al. Exercise-based rehabilitation for heart Failure. Cochrane Database Syst Rev. 2014:CD003331, http://dx.doi.org/10. 1002/14651858.CD003331.pub4./.

5. Haykowsky MJ, Daniel KM, Bhella PS, Sarma S, Kitzman DW. Heart Failure: Exercise-Based Cardiac Rehabilitation: Who. When, and How Intense? Can J Cardiol. 2016;32(10S2):S382-7, 10.1016/j.cjca.2016.06.001./.

6. Carrasquilla G, Pulido A, De la Hoz A, Mieth K, Muñoz O, Guerrero R, et al. Guía Metodológica para la elaboración de guías de práctica clínica con evaluación económica en el Sistema General de Seguridad Social en Salud Colombiano. Segunda edición, Versión completa final. Bogotá, 2014 [actualizado marzo 2014; última consulta Noviembre 2015]. Disponible en: http://gpc.minsalud.gov.co/recursos/Documents/Gu\%C3\% ADa\%20Metodológica_Web.pdf.

7. Shea BJ, Grimshaw JM, Wells GA, Boers M. Measurement tool created to assess the methodological quality of systematic reviews. BMC Medical Research Methodology. 2007;7:10.
8. QUADAS-2: A Revised Tool for the Quality Assessment of Diagnostic Accuracy Studies. Annals of Internal Medicine. 2011;155(8):529-36.

9. Guyatt GH, Oxman AD, Vist G, Kunz R, Brozek J, Alonso-Coello $P$, et al. GRADE guidelines 4: rating the quality of evidence - risk of bias. J Clin Epidemiol. 2011;64:407-15.

10. Andrews J, Guyatt G, Oxman AD, Alderson P, Dahm $P$, Falck-Ytter $Y$, et al. GRADE guidelines: 14. Going from evidence to recommendations: the significance and presentation of recommendations. J Clin Epidemiol. 2013;66:719-72, http://dx.doi.org/10.1016/j.jclinepi.2012.03.013./.

11. Guyatt G, Oxman AD, Akl EA, Kunz R, Vist G, Brozek J, et al. GRADE guidelines: 1 Introduction-GRADE evidence profiles and summary of findings tables. J Clin Epidemiol. 2011;64:383-94, http://dx.doi.org/10.1016/j.jclinepi.2010.04.026./.

12. Arena R, Myers J, Williams MA, Gulati M, Kligfield P, Balady GJ, et al. Assessment of functional capacity in clinical and research settings: a scientific statement from the American Heart Association Committee on Exercise Rehabilitation, and Prevention of the Council on Clinical Cardiology and the Council on Cardiovascular Nursing. Circulation. 2007;116:329-43.

13. Malhotra R, Bakken K, D’Elia E, Lewis GD. Cardiopulmonary Exercise Testing in Heart Failure. JACC Heart Fail. 2016;4:607-16, http://dx.doi.org/10.1016/ j.jchf.2016.03.022./.

14. Pollentier B, Irons SL, Benedetto CM, Dibenedetto AM, Loton $D$, Seyler RD, et al. Examination of the six minute walk test to determine functional capacity in people with chronic heart failure: a systematic review. Cardiopulm Phys Ther J. 2010;21:13-21.

15. Taylor RS, Sagar VA, Davies EJ, Briscoe S, Coats AJ, Dalal H, et al. Exercise-based rehabilitation for heart failure. Cochrane Database Syst Rev. 2014;4:CD003331.

16. Arena R, Myers J, Forman DE, Lavie CJ, Guazzi M. Should high-intensity-aerobic interval training become the clinical standard in heart failure? Heart Fail Rev. 2013;18: 95-105.

17. Garber CE, Blissmer B, Deschenes MR, Franklin BA, Lamonte MJ, Lee IM, et al. American College of Sports Medicine position stand. Quantity and quality of exercise for developing and maintaining cardiorespiratory, musculoskeletal, and neuromotor fitness in apparently healthy adults: guidance for prescribing exercise. Med Sci Sports Exerc. 2011;43:1334-59.

18. Smart NA, Dieberg G, Giallauria F. Intermittent versus continuous exercise training in chronic heart failure: a meta-analysis. Int J Cardiol. 2013;166:352-8.

19. Ismail H, McFarlane JR, Nojoumian AH, Dieberg G, Smart NA. Clinical outcomes and cardiovascular responses to different exercise training intensities in patients with heart failure: a systematic review and meta-analysis. JACC Heart Fail. 2013;1:514-22.

20. Smart NA. How do cardiorespiratory fitness improvements vary with physical training modality in heart failure patients? A quantitative guide. Exp Clin Cardiol. 2013;18:e21-5.

21. Patwala AY, Woods PR, Sharp L, Goldspink DF, Tan LB, Wright DJ. Maximizing patient benefit from cardiac resynchronization therapy with the addition of structured exercise training: a randomized controlled study. J Am Coll Cardiol. 2009;53: 2332-9.

22. Conraads VM, Vanderheyden M, Paelinck B, Verstreken S, Blankoff I, Miljoen $\mathrm{H}$, et al. The effect of endurance training on exercise capacity following cardiac resynchronization therapy in chronic heart failure patients: a pilot trial. Eur J Cardiovasc Prev Rehabil. 2007;14:99-106.

23. Belardinelli R, Capestro F, Misiani A, Scipione P, Georgiou D. Moderate exercise training improves functional capacity, quality of life, and endothelium-dependent vasodilation in chronic heart failure patients with implantable cardioverter 
defibrillators and cardiac resynchronization therapy. Eur J Cardiovasc Prev Rehabil. 2006;13:818-25.

24. Berg SK, Svendsen JH, Zwisler AD, Pedersen BD, Preisler P, Siersbæk-Hansen L, et al. COPE-ICD: a randomised clinical trial studying the effects and meaning of a comprehensive rehabilitation programme for ICD recipients -design, intervention and population. BMC Cardiovasc Disord. 2011;11:33.

25. Berg SK, Pedersen PU, Zwisler AD, Winkel P, Gluud C, Pedersen $\mathrm{BD}$, et al. Comprehensive cardiac rehabilitation improves outcome for patients with implantable cardioverter defibrillator Findings from the COPE-ICD randomised clinical trial. Eur J Cardiovasc Nurs. 2015;14:34-44.

26. Cowie A, Thow MK, Granat MH, Mitchell SL. Effects of home versus hospital-based exercise training in chronic heart failure. Int J Cardiol. 2012;158:296-8.
27. Karapolat H, Demir E, Bozkaya YT, Eyigor S, Nalbantgil S, Durmaz B, et al. Comparison of hospital-based versus home-based exercise training in patients with heart failure: effects on functional capacity, quality of life, psychological symptoms, and hemodynamic parameters. Clin Res Cardiol. 2009;98:635-42.

28. Daskapan A, Arikan H, Caglar N, Tunali N, Ataman S. Comparison of supervised exercise training home-based exercise training in chronic heart failure. Saudi Med J. 2005;26:842-7.

29. Davidson PM, Cockburn J, Newton PJ, Webster JK, Betihavas $\mathrm{V}$, Howes $\mathrm{L}$, et al. Can a heart failure-specific cardiac rehabilitation program decrease hospitalizations and improve outcomes in high-risk patients? Eur J Cardiovasc Prev Rehabil. 2010;17:393-402. 